\title{
Slot-Based Model for IEEE 802.15.4 MAC with Sleep Mechanism
}

\author{
Zhuoling Xiao, Chen He, Member, IEEE, and Lingge Jiang, Member, IEEE
}

\begin{abstract}
In this letter, we develop an exactly slot-based model for IEEE 802.15.4 protocol with sleep mechanism in real-time applications. By explicitly modeling the sleep mechanisms and CSMA/CA mechanism with a precision of slot, we accurately evaluate the performance of the protocol, including energy consumption and throughput. We take into consideration the impacts of several factors, including duty cycle, network traffic and initial backoff exponent. NS-2 simulations show the accuracy of the proposed model.
\end{abstract}

Index Terms-IEEE 802.15.4, sleep mechanism, CSMA/CA, energy consumption, throughput.

\section{INTRODUCTION}

$\mathbf{I}$ EEE 802.15.4 [1] has been adopted in many low complexity, ultra low power consumption, and low data rate wireless networks such as Wireless Sensor Networks (WSNs) [2] in recent years. Power consumption is always a crucial problem considered for these battery-powered nodes in such networks. To address the power consumption requirements, a simple and effective solution is to put nodes into sleep mode. This is highly effective in energy saving especially in low data rate applications such as monitoring. The detailed benefits of sleep mechanism are provided in [3].

Many model-based performance evaluations, such as [4], [5] and more recent work [6], [7], have given accurate models for CSMA/CA mechanism of IEEE 802.15.4, but they do not take the sleep mechanism into consideration. [8] has analyzed the performance of sleeping nodes in WSNs, but it neither focused on the medium access control mechanism nor evaluated the performance of IEEE 802.15.4. J. Mišić et. al have proposed an $\mathrm{M} / \mathrm{G} / 1 / \mathrm{K}$ system with vacations to analyze the sleep mechanism of a beacon-enabled IEEE 802.15.4 cluster [9]. However, the analysis focuses on the queuing aspects, while the model for the sleep mechanism assumes that a node goes to sleep for a geometrically distributed amount of time after its buffer becomes empty, which differs much from the actual mechanism and makes the analysis rather inaccurate in that aspect. Most of these works followed an approach similar to that of Bianchi's for IEEE 802.11 DCF [10] to model IEEE 802.15.4 slotted CSMA/CA.

In this letter, we propose a new slot-based analytical model for IEEE 802.15.4 taking sleep mechanism into consideration. The proposed model consists of two submodels, namely 1) a sleep model which considers the node behavior in idle and

Manuscript received October 9, 2009. The associate editor coordinating the review of this letter and approving it for publication was J. Kim.

The authors are with the Department of Electronic Engineering, Shanghai Jiaotong University, Shanghai, 200240 China (e-mail: \{zlxiao, chenhe, lgjiang\}@sjtu.edu.cn).

The research was partly supported by the National Natural Science Foundation of China Grant No. 60832009, 60872017, 60772100, and FRDC.

Digital Object Identifier 10.1109/LCOMM.2010.02.091981

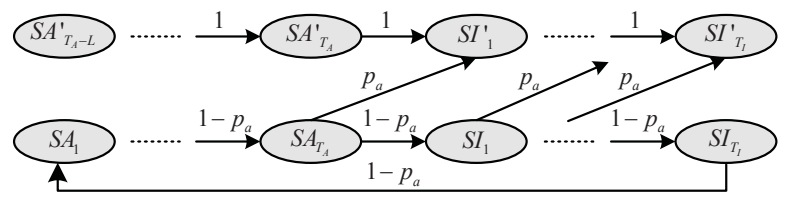

Fig. 1. Markov-based model for sleep mechanism of IEEE 802.15.4.

sleep mode; 2) a CSMA/CA model which studies the backoff mechanism of IEEE 802.15.4 CSMA/CA.

\section{AnAlytical Model Formulation}

In this study, we consider a single-hop wireless personal area network which is composed of a network coordinator and $N$ identical end nodes. The analysis is limited to realtime applications where the duty cycle is made to be large compared to the traffic load in order to reduce delay, in which case we can assume that each node buffers at most one pending data packet, and any older ones are discarded as stale data.

\section{A. Slot-based Model for Sleep Mechanism}

IEEE 802.15.4 uses a superframe structure, where each superframe is bounded by beacons periodically transmitted by coordinator, and is divided into an active period and an optional inactive period.

Fig. 1 shows the Markov-based model for sleep mechanism of IEEE 802.15.4. Our model is exactly slot-based, that is each backoff slot in a superframe is defined as a single state. The benefit of the exactly slot-based mechanism is that we can determine the interactions between the states in sleep model and CSMA/CA model with a precision of slot.

Let $p_{a}$ denote the (assumed to be constant) packet arriving probability in a single backoff slot. Since it takes 2 backoff slots for Clear Channel Assessment (CCA) and $L$ backoff slots for data packet transmission, a node can have a pending data packet waiting for transmission via CSMA/CA either in the inactive period, or in the last $L+1$ slots of the active period due to an inadequate number of slots left for CCA and transmission after backoff. Therefore, let the states $S A_{i}^{\prime}\left(i=T_{A}-L, T_{A}-\right.$ $\left.L+1, \ldots, T_{A}\right)$ and $S A_{i}\left(i=1,2, \ldots, T_{A}\right)$ stand for the sleep states with and without data packet waiting for transmission in active period of a superframe, and $S I_{i}^{\prime}, S I_{i}\left(i=1,2, \ldots, T_{I}\right)$ denote the sleep states with and without pending packet in inactive period, respectively, where $T_{A}$ and $T_{I}$ are the number of backoff slots in each active and inactive period.

More importantly, we now discuss the state transitions between sleep model and CSMA/CA model, as shown in Fig. 2.

$[\mathrm{A}],[\mathrm{B}]$, and $[\mathrm{C}]$ are the state transition interfaces between the sleep model and CSMA/CA model. As shown in 


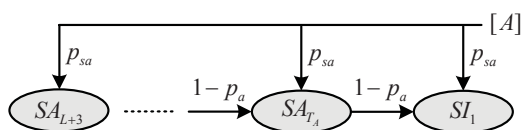

(a) State transitions after successful transmission

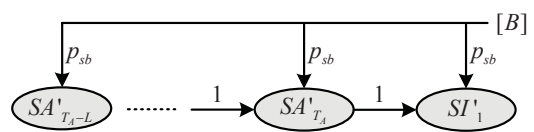

(b) State transitions after transmission failure due to inadequate slots

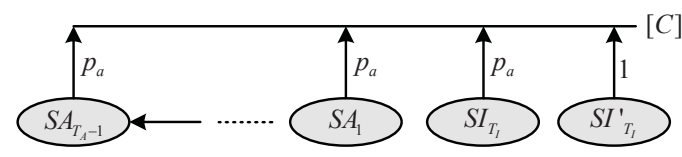

(c) State transitions after arrival of packets in active period

Fig. 2. State transitions between sleep model and CSMA/CA model.

Fig. 2a, [A] is the state transition after a successful packet transmission. Since it takes $L+2$ backoff slots for CCA and transmission, nodes cannot enter the first $L+2$ slots in active period through [A] from CSMA/CA model. As we do not keep track of the slot number in the CSMA/CA, we approximately assume that the node ends up in one of the remaining slots with equal probability $p_{s a}$.

$$
p_{s a}=1 /\left(T_{A}-L-1\right)
$$

Fig. $2 b$ shows the state transition after a failed transmission caused by inadequate slots left for CCA and data packet transmission [B]. Similarly approximated, we have

$$
p_{s b}=1 /(L+2)
$$

$[\mathrm{C}]$ is the state transition from idle state (including the last backoff slot of inactive period without pending packet) to backoff state upon packet arrival; note that packets arriving at a node with a pending one are simply discarded. Therefore, the transition probability in Fig. $2 \mathrm{c}$ is $p_{a} .[\mathrm{C}]$ also contains the transition from the last backoff slot of inactive period with pending packet to backoff states with probability 1 .

In order to solve the model, we still need the steady state probabilities of a node going through [A] (resp. [B]) per slot, denoted by $\pi_{A}$ and $\pi_{B}$ ( $\pi_{S}$ stands for the steady state probability of $S$ in this letter), respectively. From Fig. 2a, 2b, and Fig. 3 which shows the Markov model for CSMA/CA mechanism in IEEE 802.15.4, we can have

$$
\pi_{A}=\pi_{T_{L}}, \pi_{B}=\gamma \phi
$$

where $T_{L}, \gamma$, and $\phi$ are defined in the analysis of CSMA/CA model.

\section{B. Model for CSMA/CA Mechanism}

As shown in Fig. 3, The Markov model for CSMA/CA mechanism in IEEE 802.15.4 is extended from [10]. Let state $B O_{i, j}\left(i=0,1, \ldots, M ; j=0,1, \ldots, W_{i}-1\right)$ be the $j$ th backoff state in the $i$ th backoff stage. $M$ is the maximum number of backoff stage. $W_{i}$ is the maximum number of backoff slots in $i$ th backoff stage. State $C C A_{i, j}(i=0,1, \ldots, M ; j=0,1)$ denotes the $j$ th CCA state in the $i$ th backoff stage. State $T_{i}(i=1,2, \ldots, L)$ is the $i$ th transmitting state.

The transition probabilities $\alpha$ and $\beta$ stand for the probabilities that the channel is assessed to be busy during the first CCA and during the second one provided that the first reported idle, respectively. They can be derived in a similar way in [4] as

$$
\alpha=L\left[1-(1-\phi(1-\gamma))^{N-1}\right](1-\alpha)(1-\beta)
$$

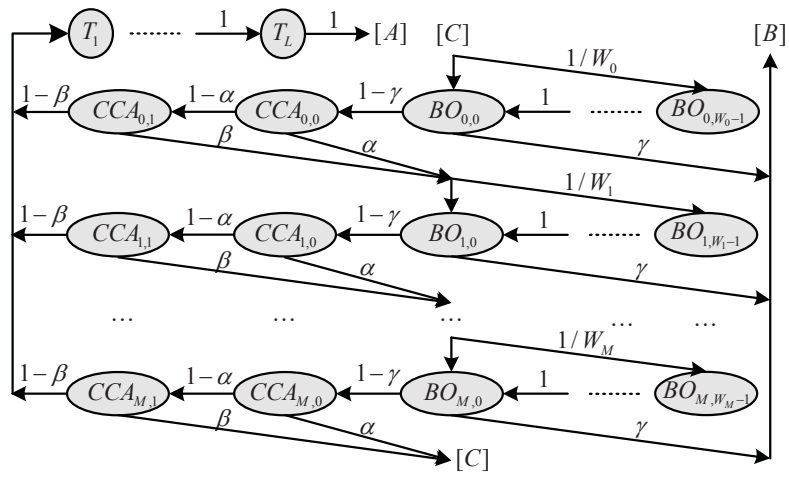

Fig. 3. Markov-based model for slotted CSMA/CA mechanism of IEEE 802.15.4.

$$
\beta=\left[1-(1-\phi(1-\gamma))^{N-1}\right]\left[2-(1-\phi(1-\gamma))^{N}\right]^{-1}
$$

in which $\phi=\sum_{i=0}^{M} \pi_{B O_{i, 0}} \cdot \gamma$ is the transmission failure probability due to an insufficient number of slots left in current active period. Since it costs $L+2$ backoff slots for CCA and transmission, this probability can be approximated as

$$
\gamma=(L+2) / T_{A}
$$

According to Fig. 2c, The steady state probability of interface $[C]$ is

$$
\pi_{C}=\pi_{S I_{T_{I}}^{\prime}}+p_{a}\left(\sum_{i=1}^{T_{A}} \pi_{S A_{i}}+\pi_{S I_{T_{I}}}\right)
$$

\section{Model Validation}

The proposed model is validated by the ns- 2.33 simulator. Simulation parameters are set as follows: data packet payload, MAC header, and PHY header are 80 bytes, 21 bytes and 6 bytes, respectively. Transmission rate is $250 \mathrm{kbps}$. The power parameters are the same as Chipcon CC2420. Buffer size is set to be 1 and $T_{A}+T_{I}=2^{6}$. Other simulation parameters are the same as their default values defined in the standard [1].

\section{A. Energy Consumption Analysis}

In energy consumption analysis, we also take into account several other factors including the transmitting/receiving of acknowledgement packets and periodical beacons, and mode transition from receiving mode to transmitting mode after twice CCA if the node is ready to transmit.

Therefore, the average energy consumption of each packet $E_{p}$ can be defined as follows:

$$
E_{p}=\left(E_{t x}+E_{r x}+E_{\text {idle }}+E_{\text {sleep }}\right) / N_{p}
$$

where $N_{p}$ is the average number of packets transmitted by each node per second; $E_{t x}, E_{r x}, E_{\text {idle }}$, and $E_{\text {sleep }}$ are the 

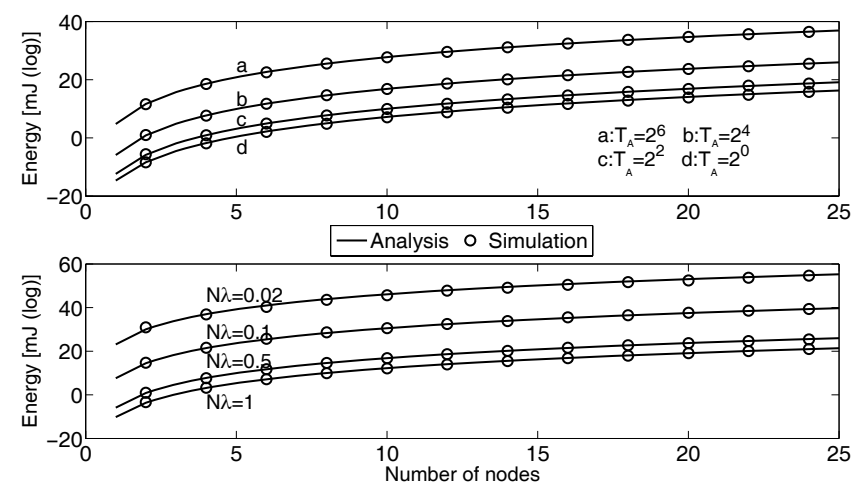

Fig. 4. Energy consumption of each packet versus number of nodes for different duty cycles $(N \lambda=0.5)$ and different network loads $\left(T_{A}=2^{4}\right)$.

energy consumed in a second in transmitting, receiving, idle, and sleep states of each node, respectively. They are defined as follows:

$$
\begin{aligned}
& E_{t x}=P_{t x} \pi_{T} \\
& E_{r x}=P_{r x}\left((N-1) \pi_{T}+\pi_{C C A}+p_{b r t}\right)+E_{A C K} \\
& E_{\text {idle }}=P_{\text {idle }}\left(\pi_{S A}+\pi_{S A^{\prime}}+\pi_{B O}-p_{b r t}\right) \\
& E_{\text {sleep }}=P_{\text {sleep }}\left(\pi_{S I}+\pi_{S I^{\prime}}\right) \\
& E_{A C K}=P_{r x} N \pi_{T} L_{A C K} / L \\
& p_{\text {brt }}=\left(T_{\text {beacon }}+T_{r t}\right) /\left(T_{A}+T_{I}\right)
\end{aligned}
$$

where $P_{t x}, P_{r x}, P_{\text {idle }}, P_{\text {sleep }}$ are the power consumption of transmitting, receiving, idle, and sleep states, respectively.

Fig. 4 compares the predicted and simulated energy consumption per packet. $\lambda$ is the packet arrival rate of the network provided that the number of packets is Poisson distributed. It can be observed that the increase in duty cycle and decrease in network load will both result in the increase in energy consumption. Meanwhile, the energy consumption per packet goes up as number of nodes increases. These characteristics are obvious in practical applications. Our model analysis fits well with the simulation results.

\section{B. Throughput Analysis}

According to the preceding CSMA/CA model, the average number of slots spent in backoff states per packet, denoted by $B$, should satisfy the following equation:

$$
B=\sum_{i=0}^{M} \frac{q^{i}\left(W_{i}+1\right)}{2}+\phi \gamma\left(\frac{L+2}{2}+T_{I}+B\right)
$$

where $q=(1-\gamma)[\alpha+(1-\alpha) \beta]$. So the total number of slots $D$ that a node spends serving a packet in average should be

$$
D=B+L+2
$$

Therefore, the saturated throughput, i.e. the maximum number of packets that can be successfully transmitted during a single active period for each node, can be calculated by

$$
S=T_{A}(D N)^{-1}
$$

Fig. 5 shows the impact of duty cycle and initial backoff exponent on throughput of each node. From (12), the increase
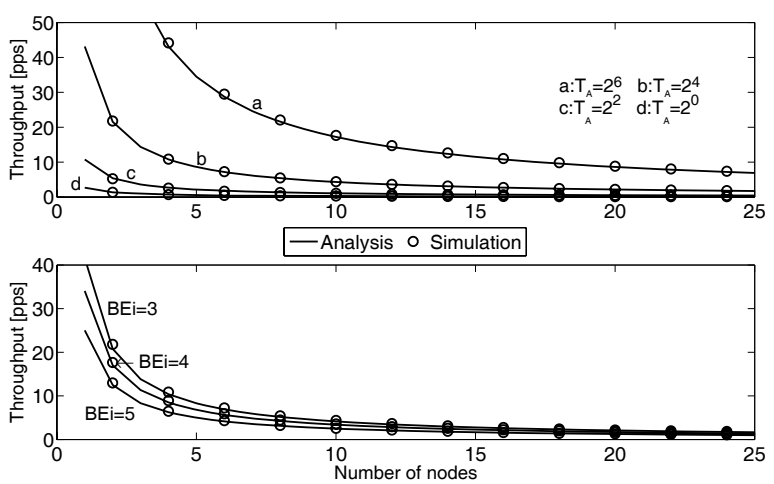

Fig. 5. Throughput of each node versus number of nodes for different duty cycle $(N \lambda=0.5)$ and different initial backoff exponent $\left(T_{I}=2^{4}, N \lambda=\right.$ $0.5)$.

in duty cycle will increase the throughput. While from (10) with the same number of nodes in the network, the increase of the initial backoff exponent results in the increase of backoff time, and thus the decrease of throughput of each node.

\section{Conclusions}

We have proposed a new analytical model for IEEE 802.15.4 MAC with sleep mechanism to compute the energy consumption and throughput in single-hop networks in realtime applications. Because the submodel for sleep mechanism is exactly slot-based, the accuracy of the whole model can be guaranteed. The simulation results show that the proposed model can accurately evaluate the performance of IEEE 802.15.4 in real-time applications.

\section{REFERENCES}

[1] IEEE TG 15.4, Part 15.4: Wireless Medium Access Control (MAC) and Physical Layer (PHY) Specifications for Low-Rate Wireless Personal Area Networks (WPANs), IEEE Std., New York, 2006.

[2] J. Zheng and M. J. Lee, "Will IEEE 802.15.4 make ubiquitous networking a reality? A discussion on a potential low power, low bit rate standard," IEEE Commun. Mag., vol. 42, no. 6, pp. 140-146, June 2004.

[3] S. Singh and C. S. Raghavendra, "PAMAS: power aware multi-access protocol with signaling for ad hoc networks," ACM Computer Commun. Rev., vol. 28, no. 3, pp. 5-26, July 1998.

[4] T. R. Park, T. H. Kim, J. Y. Choi, et al., "Throughput and energy consumption analysis of IEEE 802.15.4 slotted CSMA/CA," Electron. Lett., vol. 41, no. 18, pp. 1017-1019, Sept. 2005.

[5] Y. Zhang, P. Xu, Z. Zhang, et al., "Comments on throughput analysis of IEEE 802.15.4 slotted CSMA/CA considering timeout period," IEEE Electron. Lett., vol. 42, no. 19, pp. 1127-1128, Sept. 2006.

[6] S. Pollin, M. Ergen, S. C. Ergen, et al., "Performance analysis of slotted carrier sense IEEE 802.15.4 medium access layer," IEEE Trans. Wireless Commun., vol. 7, no. 9, pp. 3359-3371, Sept. 2008.

[7] J. He, Z. Tang, H. H. Chen, et al., "An accurate markov model for slotted CSMA/CA algorithm in IEEE 802.15.4 networks," IEEE Commun. Lett., vol. 12 , no. 6 , pp. 420-422, June 2008.

[8] C. F. Chiasserini and M. Garetto, "An analytical model for wireless sensor networks with sleeping nodes," IEEE Trans. Mob. Comp., vol. 5, no. 12, pp. 1706-1718, Dec. 2006.

[9] J. Mišić and V. B. Mišić, "Queuing analysis of sleep management in an 802.15.4 beacon enabled PAN," in Proc. IEEE/ACM First International Workshop on Broadband Wireless Services and Applications (BroadWISE '04), San Jose, CA, Oct. 2004.

[10] G. Bianchi, "Performance analysis of the IEEE 802.11 distributed coordination function," IEEE J. Sel. Areas Commun., vol. 18, no. 3, pp. 535-547, Mar. 2000. 